TRANSACTIONS OF THE

AMERICAN MATHEMATICAL SOCIETY

Volume 355, Number 2, Pages 619-636

S 0002-9947(02)03162-8

Article electronically published on October 9, 2002

\title{
TEST IDEALS AND BASE CHANGE PROBLEMS IN TIGHT CLOSURE THEORY
}

\author{
IAN M. ABERBACH AND FLORIAN ENESCU
}

\begin{abstract}
Test ideals are an important concept in tight closure theory and their behavior via flat base change can be very difficult to understand. Our paper presents results regarding this behavior under flat maps with reasonably nice (but far from smooth) fibers. This involves analyzing, in depth, a special type of ideal of test elements, called the CS test ideal. Besides providing new results, the paper also contains extensions of a theorem by G. Lyubeznik and K. E. Smith on the completely stable test ideal and of theorems by F. Enescu and, independently, M. Hashimoto on the behavior of $F$-rationality under flat base change.
\end{abstract}

\section{INTRODUCTION AND TERMINOLOGY}

Let $R$ be a Noetherian commutative ring of positive prime characteristic $p$. Over the last decade tight closure theory has played a tremendous role in understanding the structure of $R, 9]-12$, [16. One compelling problem is how tight closure behaves under flat base change. This problem has multiple facets and it is intimately connected to the problem of localization of tight closure, as shown by M. Hochster and $\mathrm{C}$. Huneke in [11. In fact, they show that even the case when the fibers are "good" (as in the case of smooth base change) is very complicated and not entirely understood. Several important questions remain open even in the case of the completion morphism of a local excellent ring. With this in mind, the goal of understanding how tight closure behaves under flat base change, while weakening the smoothness conditions on the fibers, is quite challenging.

The key idea has become to allow a wider class of fibers. Recently techniques have been developed to better understand how $F$-rationality, weak $F$-regularity, and strong $F$-regularity behave under certain flat local homomorphisms $(R, \mathbf{m}) \rightarrow(S, \mathbf{n})$ with non-smooth fibers ([3], 7], [8]). It is now apparent that significant progress can be obtained in studying the tight closure of $\mathbf{m}$-primary ideals for flat local homomorphisms (as the results in 3 indicated). At the same time, Lyubeznik and Smith initiated a new approach to handle the localization and completion problem for certain test ideals [16]. Test elements are a crucial tool in tight closure theory and the question of how they behave under localization is one of the central problems of the theory. In fact, the knowledge that $1 \in R$ is a test element is not (yet) known

Received by the editors October 30, 2001.

2000 Mathematics Subject Classification. Primary 13A35, Secondary 13B40.

The first author was partially supported by the NSF and by the University of Missouri Research Board. The second author thanks the University of Michigan for support through the Rackham Predoctoral Fellowship. 
to imply that $1 \in R_{P}$ is a test element! Lyubeznik and Smith suggested that one should understand first the case of a particular ideal contained in the test ideal (and conjecturally equal to it in the case of excellent reduced rings). In the local reduced case, this ideal, $\widetilde{\tau}(R)$, is the annihilator of the tight closure of 0 in the injective hull of the residue field. They show that $\widetilde{\tau}(R)$ (called here the $C S$ test ideal) behaves as expected with respect to localization and completion for rings that are images of excellent regular local rings. Although able to prove the preceding statement, Lyubeznik and Smith expected this to be true for all excellent local rings (see the first paragraph after Theorem 7.1 in [16]).

In our paper we investigate the behavior of tight closure of ideals primary to the maximal ideal (in local excellent reduced rings) under flat base change. Our work relies on the CS test ideal and its properties. The conditions on the fibers of the homomorphism are greatly weakened and our results apply in many situations other than that of ideals primary to the maximal ideal. We explore this in section 4. However, as we just said, we must develop several theorems about test elements which, in fact, are interesting in their own right. These appear in section 3. Along the way we show that the CS test ideal respects both completion and localization in excellent reduced local rings, as Lyubeznik and Smith expected. Extending their result to the class of excellent rings is more than a technical achievement. On one hand, J. Nishimura has given examples of excellent rings that are not images of regular rings (see [18]). On the other hand, while much of tight closure theory has been shown to apply well to the class of excellent rings, recent examples of Loepp and Rotthaus show that if one goes beyond this class, then tight closure does not even commute with completion, [15]. Our theorem provides more evidence in support of the belief that tight closure behaves well in excellent rings. To prove the theorem we make use of Lyubeznik's and Smith's result that the CS test ideal localizes in complete local reduced rings. The reduction is done by the very means of flat base change, yet in a non-canonical manner. It underlines the theoretical importance of results on the behavior of tight closure under such base change and highlights how understanding even smooth base change depends on understanding flat base change where the fibers are not regular.

This result appears below as:

Theorem 3.6. Let $(S, \underline{\mathbf{n}})$ be a semi-local reduced excellent ring. Then

(1) $\widetilde{\tau}(S)$ localizes properly at any prime $Q \in \operatorname{Spec}(S)$, i.e., $\widetilde{\tau}(S)_{Q}=\widetilde{\tau}\left(S_{Q}\right)$.

(2) $\widetilde{\tau}(\widehat{S})=\widetilde{\tau}(S) \widehat{S}$.

(3) Let $(R, \mathbf{m})$ be a semi-local reduced excellent ring such that $(R, \underline{\mathbf{m}}) \rightarrow(S, \underline{\mathbf{n}})$ is a flat semi-local map with Gorenstein F-injective closed fibers and such that if $\mathbf{n}$ is a maximal ideal of $S$ lying over $\mathbf{m}$, then $R / \mathbf{m} \rightarrow S / \mathbf{n}$ is separable. Assume that $R$ and $S$ have a common CS test element. Then $\widetilde{\tau}(S)=\widetilde{\tau}(R) S$.

From this we obtain that the CS test ideal extends properly in a number of good cases:

Corollary 3.8. Let $(R, \mathbf{m}) \rightarrow(S, \mathbf{n})$ be a flat map of reduced local rings such that $S / \mathbf{m} S$ is Gorenstein and $0_{E_{S}}^{*}$ is extended. Then $\widetilde{\tau}(S)=\widetilde{\tau}(R) S$.

It is clear that test elements are important in problems of base change. Our investigation also shows that it suffices to have available a particular type of test 
elements, namely the CS test elements. Generally, these elements are as abundant as the test elements and one now has the advantage that the theory of the CS test ideal is well understood (as our results just quoted above, together with those of Lyubeznik and Smith, show).

In section 4 we turn to the question of extending tight closure by flat base change. One of the main cases where our results apply is that of ideals primary to the maximal ideal in local excellent rings. We extend many of the known results. To be precise, Hochster and Huneke ([11]) have results on the behavior of tight closure of ideals under smooth (or regular, using a more often used terminology) base change; we extend their results by weakening the conditions on the fibers. Aberbach ([3]) has results on the behavior of weak $F$-regularity obtained by considering the behavior of irreducible ideals; in contrast, we obtain results for all ideals primary to the maximal ideal. These results are considerably more difficult, since non-irreducible ideals have larger dimension socles, which turn out to require new ideas for carrying out tight closure arguments. Enescu ([7]) and, independently, Hashimoto ([] $)$ have results on the behavior of $F$-rationality under some finiteness conditions (their theorems in turn extend Vélez's results on F-rationality, [20]); ours show that these conditions are not necessary. A feature of the study is that some of the tools on which we rely heavily are as basic as Nakayama's Lemma.

We would like to illustrate some of our results here. For example, Theorem 4.1 shows that tight closure extends well when the closed fiber is Gorenstein (and either $F$-injective or $F$-rational). However, when the closed fiber is assumed to be only $\mathrm{CM}$, the arguments have been more difficult. We have been able to show:

Theorem 4.2. Let $(R, \mathbf{m}, K) \rightarrow(S, \mathbf{n}, L)$ be a flat map of excellent rings with $C M$ closed fiber. Let $I \subseteq R$ be $\mathbf{m}$-primary and tightly closed. Let $\mathbf{z}=z_{1}, \ldots, z_{d}$ be elements whose image in $S / \mathbf{m} S$ is an s.o.p. Suppose that either

(1) the element $c \in R^{\circ}$ is a common test element for $R$ and $S$, and that $S / \mathbf{m} S$ is geometrically $F$-injective over $K$, or

(2) $S / \mathbf{m} S$ is geometrically $F$-rational over $K$.

Then $(I, \mathbf{z}) S$ is tightly closed.

When $R \rightarrow S$ is a ring homomorphism, there is a large number of results in the literature that involve the need for a common test element (see for example our Theorem 3.3). This is a somewhat restrictive requirement, and even when there is such a common test element, its existence may not be known a priori. Section 5 deals with this issue. We use the notion of strong test ideals to show how this assumption can be removed in certain conditions (that are more general than the ones in 11], see for example Discussion 7.11) and still have that tight closure behaves well:

Theorem 5.3. Let $(R, \mathbf{m}, K) \rightarrow(S, \mathbf{n}, L)$ be a flat map of excellent domains both of whose completions are domains. Assume that $S / \mathbf{m} S$ is Gorenstein and F-rational and $K \rightarrow L$ is separable. Then $0_{E_{S}}^{*}$ is extended from $0_{E_{R}}^{*}$, and $\widetilde{\tau}(S)=\widetilde{\tau}(R) S$.

Also, $0_{E_{S}}^{* f g}$ is extended from $0_{E_{R}}^{* f g}$ and hence $R$ and $S$ have a common test element.

In fact, we prove results that show the existence of CS test elements for $S$ of a convenient form relative to $R$ (Theorems 3.9 and 5.6). It can be argued that they are mainly of technical significance. However, many of the theorems in section 4 make use of such ideas. We believe that they will prove to be useful in a number of contexts different from ours. 
In the last section of the paper we give results concerning Hilbert-Kunz multiplicities and test exponents.

We now define tight closure and related concepts (see [9] and [11]). We use $q$ to denote a power of $p$; so $q=p^{e}$ for $e \geq 0$. For $I \subseteq R$ set $I^{[q]}=\left(i^{q}: i \in I\right)$. Let $R^{\circ}$ be the complement in $R$ of the minimal primes of $R$. We say that $x \in I^{*}$, the tight closure of $I$, if there exists $c \in R^{\circ}$ such that for all $q \gg 0, c x^{q} \in I^{[q]}$. We say that $x$ is in the Frobenius closure of $I, I^{F}$, if there exists a $q$ such that $x^{q} \in I^{[q]}$, and say that $I$ is Frobenius closed if $I=I^{F}$. When $R$ is reduced, then $R^{1 / q}$ denotes the ring of $q$ th roots of elements of $R$. Note that in this case, $c x^{q} \in I^{[q]}$ if and only if $c^{1 / q} x \in I R^{1 / q}$. When $R^{1 / q}$ is module-finite over $R$, we call $R F$-finite. We call $R$ weakly $F$-regular if every ideal of $R$ is tightly closed. A weakly $F$-regular ring is always normal, and under mild hypotheses is Cohen-Macaulay. $R$ is $F$-regular if every localization of $R$ is weakly $F$-regular. An $F$-finite reduced ring $R$ is called strongly F-regular if for all $c \in R^{\circ}$ there exists $q$ such that $R c^{1 / q} \subseteq R^{1 / q}$ splits over $R$. Strongly $F$-regular implies $F$-regular. It is open in most cases whether or not these notions are equivalent.

We call an ideal $I=\left(x_{1}, \ldots, x_{n}\right)$ a parameter ideal if ht $I \geq n . R$ is $F$-rational if every parameter ideal is tightly closed. $F$-rational and Gorenstein rings are (strongly) $F$-regular. We call the local ring $(R, \mathbf{m}) F$-injective if the Frobenius endomorphism $R \rightarrow R$ sending $r \mapsto r^{p}$ induces an injection on all the local cohomology modules of $R$. When $R$ is CM, then $R$ is $F$-injective if and only if some (equivalently, every) ideal generated by a system of parameters is Frobenius closed.

There is a tight closure operation for a submodule $N \subseteq M$. Let $R^{(e)}$ be the $R$-bimodule whose underlying group structure is the same as for $R$, considered as a left $R$-module in the usual way, but as a right $R$-module via the $e$ th iteration of the Frobenius map sending $r \mapsto r^{p^{e}}$. Then $F_{R}^{e}(M)=R^{(e)} \otimes_{R} M$ considered as a left $R$-module. For $m \in M$, we let $m^{q}=1 \otimes m \in F_{R}^{e}(M)$. If $N \subseteq M$, then we let $N_{M}^{[q]}$ be the image of $F_{R}^{e}(N)$ in $F_{R}^{e}(M)$. An element $x \in M$ is in the tight closure of $N$ in $M$, denoted $N_{M}^{*}$ if there exists $c \in R^{\circ}$ such that for all $q \gg 0, c x^{q} \in N_{M}^{[q]}$. The finitistic tight closure of $N$ in $M$, denoted $N_{M}^{* f g}$ is the union of all $\left(N \cap M^{\prime}\right)_{M^{\prime}}^{*}$ where the union is taken over all finitely generated submodules $M^{\prime} \subseteq M$.

We will often be considering modules of the form $M=\lim _{t} R / I_{t}$ for a sequence of ideals $\left\{I_{t}\right\}$. Thus it is useful to discuss tight closure in this case. Let $u \in M$ be an element which is given by $\left\{u_{t}\right\}$ where in the direct limit system $u_{t} \mapsto u_{t+1}$. Then $u \in 0_{M}^{*}$ if there exists $c \in R^{\circ}$ and a sequence $t_{q}$ such that for all $q \gg 0$, $c u_{t_{q}}^{q} \in I_{t_{q}}^{[q]}$, and $u$ is in $0_{M}^{* f g}$ if there exists $c \in R^{\circ}$ and $t>0$ such that $c u_{t}^{q} \in I_{t}^{[q]}$ for all $q$. Clearly $0_{M}^{* f g} \subseteq 0_{M}^{*}$.

The element $c \in R^{\circ}$ is a test element if whenever $x \in I^{*}$ (for any $I$ ), then $c x^{q} \in I^{[q]}$ for all $q$. The element $c \in R^{\circ}$ is a completely stable test element if $c$ is a test element in every completion of every localization of $R$. When $R$ is an excellent reduced local ring, then $R$ has an abundance of test elements. When $R$ is excellent and reduced, which is the only case we will consider here, then any (completely stable) test element for ideals is also a (completely stable) test element for tight closure in finitely generated modules. Of course, we will be very interested in tight closure in non-finitely generated modules such as the injective hull of the residue field. We deal with this situation in section 2 . 
Theorem 1.1 ([1], Theorem 6.2). Let $(R, m)$ be a reduced excellent local ring. If $c \in R^{\circ}$ and $R_{c}$ is Gorenstein and F-regular, then $c$ has a power which is a completely stable test element.

One way to understand the singularities of $R$ is to understand the test ideal (respectively, the completely stable test ideal). The test ideal of $R$ is $\tau(R)=\{c \in$ $R$ : for all $I$ and all $q, x \in I^{*}$ if and only if $\left.c x^{q} \in I^{[q]}\right\}$. The completely stable test ideal is defined analogously. Suppose that $(R, \mathbf{m})$ is reduced and approximately Gorenstein, i.e., there exists a sequence $\left\{I_{t}\right\}$ of $\mathbf{m}$-primary irreducible ideals cofinal with the powers of $\mathbf{m}$. Then $\tau(R)=\bigcap_{t}\left(I_{t}: I_{t}^{*}\right)$. If $E_{R}=E_{R}(R / \mathbf{m})$ is the injective hull of the residue field, then we also have $\tau(R)=\operatorname{Ann}_{R} 0_{E_{R}}^{* f g}$.

We would like to thank the referee for useful editorial suggestions and Graham Leuschke for providing us a copy of the preprint [18].

\section{CS TEST ELEMENTS}

As mentioned earlier, Lyubeznik and Smith have defined the ideal $\widetilde{\tau}(R)=$ $\operatorname{Ann}_{R} 0_{E_{R}}^{*}$, which is contained in the completely stable test ideal (and is probably equal to it when $R$ is a reduced excellent local ring).

Definition. We will call $\widetilde{\tau}(R)$ the $C S$ test ideal of $R$. The elements of $\widetilde{\tau}(R)$ that are in $R^{\circ}$ will be called $C S$ test elements.

Remember that $\tau(R)=\operatorname{Ann}_{R} 0_{E_{R}}^{* f g}$. It is conjectured, but open in most cases, that in fact $0_{E_{R}}^{*}=0_{E_{R}}^{* f g}$. Clearly, $\widetilde{\tau}(R) \subseteq \tau(R)$.

When $R$ is not local, we will use the convention in [16] that $E_{R}=\bigoplus_{\mathrm{m}} E_{R_{\mathrm{m}}}$ where the sum is over all maximal ideals of $R$. It is natural to ask if given a multiplicative system $U \subseteq R$, we have $\tau\left(U^{-1} R\right)=U^{-1} \tau(R)$ (or if $\widetilde{\tau}\left(U^{-1} R\right)=U^{-1} \widetilde{\tau}(R)$ ). The latter equality is obtained for $R$, a reduced ring that is a homomorphic image of an excellent regular ring in [16. We show that in fact this equality holds for all excellent reduced local rings (see Theorem 3.6). Understanding localization of $\tau(R)$ is actually a more difficult task since knowing $\tau(R)$ localizes will imply that weak $F$-regularity is equivalent to $F$-regularity.

As mentioned above, understanding the (finitistic) tight closure of 0 in $E=$ $E_{R}(R / \mathbf{m})$ for the local ring $(R, \mathbf{m})$ is very important. When $(R, \mathbf{m})$ is excellent and reduced, then $E=\lim _{t} R / I_{t}$ where the $I_{t}$ are irreducible $\mathbf{m}$-primary ideals cofinal with the powers of $\mathbf{m}$. Thus the (finitistic) tight closure of 0 in $E$ may be understood as described above.

Proposition 2.1 below shows that CS test elements are precisely those elements that test tight closure in Artinian modules. Without loss of generality, it is enough to show that all such elements need work only in tight closure tests for $0_{M}^{*}$.

Proposition 2.1. Let $(R, \mathbf{m}, k)$ be an excellent reduced ring, and let $M$ be an Artinian R-module. Then

(1) $F^{e}(M)$ is Artinian for all e, and

(2) if $c \in \widetilde{\tau}(R) \cap R^{\circ}$, then $c$ works in all tight closure tests for $0_{M}^{*}$.

Proof. (1) We may assume that $R$ is complete. We can then choose $\Gamma$ such that $S=R^{\Gamma}$ is still reduced. Note that an $R$-module $N$ is Artinian over $R$ if and only if $N \otimes_{R} S$ is Artinian over $S$. Also, $F_{R}^{e}(M) \otimes_{R} S=F_{S}^{e}\left(M \otimes_{R} S\right)$; so without loss of generality we may assume that $R$ is $F$-finite. However, in general, if $R \rightarrow S$ is 
module finite, then $N$ is Artinian over $R$ if and only if $N \otimes_{R} S$ is Artinian over $S$. In this case, we are taking $S=R^{(e)}$.

(2) Suppose that $M$ is Artinian and $u \in N_{M}^{*}$. Since $M / N$ is Artinian, we may assume that $N=0$. Then $u^{q} \in 0_{F^{e}(M)}^{*}$ for all $e$. By part $(1), F^{e}(M)$ is Artinian, hence $F^{e}(M) \subseteq E^{n_{e}}$ (where $E=E_{R}(k)$ ). (To see this it is enough to tensor with $\widehat{R}$.) The image of $u^{q}$ is in the tight closure of 0 in $E^{n_{e}}$. Thus $c u^{q}=0$.

Observation. The argument in Proposition 2.1 shows that, in fact, elements of $\widetilde{\tau}(R)$ test tight closure in any module $M$ supported only on $\{\mathbf{m}\}$.

The test elements shown to exist in Theorem 1.1 are actually CS test elements. We refer the reader to section 6 of [11] for a full development of $\Gamma$-extensions. The main point is that if $R$ is a complete local ring, then one may extend the coefficient field to obtain a map $R \rightarrow R^{\Gamma}$ which is faithfully flat and purely inseparable and such that $R^{\Gamma}$ is $F$-finite. Moreover, if $R$ is reduced, then $\Gamma$ may be chosen so that $R^{\Gamma}$ is reduced.

Lemma 2.2. Let $(R, \mathbf{m}, k)$ be an excellent reduced ring. If $R_{c}$ is Gorenstein and weakly F-regular, then c has a power which is a CS test element.

Proof. We already know that $c$ has a power (which we will rename as $c$ ) which is a test element by Theorem 1.1. In fact, the proof, which involves looking at $\widehat{R}^{\Gamma}$ for sufficiently small $\Gamma$, along with Proposition 2.1, shows the CS property. Because $\widehat{R}^{\Gamma}$ is faithfully flat over $R$, it is enough to consider the case when $R$ is $F$-finite. If $E_{R}(k)=\lim _{t} R / I_{t}$ and $u=\left\{u_{t}\right\} \in 0_{E}^{*}$, then there exists $d \in R^{\circ}$ such that for all $q$ there exists $t$ such that $d u_{t}^{q} \in I_{t}^{[q]}$. For $R$ there is a $q_{0}$ and a linear map of $R^{1 / q_{0}} \rightarrow R$ sending $d^{1 / q_{0}} \rightarrow c$. Since $d u_{t}^{q q_{0}} \in I_{t}^{\left[q q_{0}\right]}$ we may take $q_{0}^{t h}$ roots and apply the splitting to see that $c u_{t}^{q} \in I_{t}^{[q]}$. Thus $c \in \operatorname{Ann}_{R} 0_{E}^{*}$.

Theorem 2.3 (see [11, Discussion 7.11). Let $(R, \mathbf{m}, k) \rightarrow(S, \mathbf{n}, L)$ be a flat map of complete local rings with regular closed fiber. Assume also that $k \rightarrow L$ is separable. Then $R$ and $S$ have a common $C S$ test element.

Proof. In light of Lemma 2.2, it is enough to find an element $c \in R$ such that $S_{c}$ is regular. We will follow 7.11 in [11]. As is done there, take $K$ to be a coefficient field for $R, \mathbf{x}=x_{1}, \ldots, x_{n}$ a system of parameters in $R$ such that $R$ is module finite over $A=K[[\mathbf{x}]]$. Let $\mathbf{y}=y_{1}, \ldots, y_{d}$ be a regular system of parameters for $S / \mathbf{m} S$ and enlarge $k$ to a coefficient field $L$ of $S$ (we can do this because $L$ is separable over $k$ ). It can be shown, as in the reference quoted above, that $S=B \otimes_{A} R$ where $B=L[[\mathbf{x}, \mathbf{y}]]$. Now it is clear that if $c \in R$ is such that $R_{c}$ is regular, then $S_{c}$ is regular (the base change from $R$ to $S$ has regular fibers). Now, Lemma 2.2 applies and we have a common CS test element for $R$ and $S$.

We end this section by stating a result that relates $E_{S}$ to $E_{R}$ for a flat local homomorphism $(R, \mathbf{m}) \rightarrow(S, \mathbf{n})$ with Gorenstein closed fiber. Our results rely heavily on this structure theorem.

Theorem 2.4 ([11]). Suppose that $(R, \mathbf{m}) \rightarrow(S, \mathbf{n})$ is a flat local homomorphism such that $S / \mathbf{m} S$ is Gorenstein. If $\mathbf{z}$ is a regular sequence for the closed fiber, then $E_{S}=\lim _{t} E_{R} \otimes_{R} S /(\mathbf{z})^{[t]}$. 


\section{§3. Completely stable test ideals under flat Base Change}

The main theorem in this section, Theorem 3.6, shows that if $(R, \mathbf{m}) \rightarrow(S, \mathbf{n})$ is a flat map of excellent reduced rings with sufficiently nice closed fiber, then the CS test ideal of $S$ is extended from $R$. It also shows that for any reduced excellent (semi-)local ring, the completely stable test ideal localizes properly.

We also wish to highlight Theorem 3.9. There are a number of results in the literature concerning a (flat) map $R \rightarrow S$ where one assumption is that $R$ and $S$ have a common test element. This assumption can be somewhat restrictive. Theorem 3.9 shows that $S$ can have useful test elements in relation to $R$ even if there is no common test element. We will make extensive use of this result in section 4 .

In the remainder of this paper we will make frequent use of the following facts 17]:

(1) If $R \rightarrow S$ is flat, $N \subseteq M$ are finitely generated $R$-modules, and $w \in M$, then $(N \otimes S):_{S}(w \otimes 1)=\left(N:_{R} w\right) \otimes S$.

(2) If $(R, \mathbf{m}) \rightarrow(S, \mathbf{n})$ is a flat local map of local rings and $\mathbf{z}=z_{1}, \ldots, z_{d} \in S$ is a regular sequence on $S / \mathbf{m} S$, then $R \rightarrow S /(\mathbf{z}) S$ is flat.

Lemma 3.1. Let $(R, \mathbf{m}) \rightarrow(S, \mathbf{n})$ be a flat map of reduced local rings with $F$ injective CM closed fiber and a common CS test element c. Suppose $\mathbf{z}=z_{1}, \ldots, z_{d}$ is an s.o.p. in $S / \mathbf{m} S$ and $b$ maps to a socle element in $S /(\mathbf{m}, \mathbf{z}) S$. If $M$ is an Artinian module and $u$ is not in $0_{M}^{*}$, then bu is not in $0_{M \otimes S /(\mathbf{z})}^{*}$.

Proof. Let $M^{\prime}=M \otimes S /(\mathbf{z})$. If the lemma is not true, then for all $q, c(b u)^{q}=0$ in $F_{S}^{e}\left(M^{\prime}\right)$. Thus $b^{q} \in 0:_{S} c u^{q}=\left(0:_{R} c u^{q}\right) S+(\mathbf{z})^{[q]} S$, by flatness. Since $u \notin 0_{M}^{*}$, $\left(0: R c u^{q}\right) \subseteq \mathbf{m}$ for infinitely many $q$. Thus $b^{q} \in(\mathbf{z})^{[q]}(S / \mathbf{m} S)$, contradicting the $F$-injectivity of $S / \mathbf{m} S$.

Remark 3.2. Let $(A, \mathbf{m}) \rightarrow(B, \mathbf{n})$ be a flat map of local rings such that $\operatorname{dim} A=0$. The map is then faithfully flat. In this situation $B$ is a free $A$-module and any basis of $B / \mathbf{m} B$ lifts to a free basis of $B$ over $A$. To see this, let $\left\{\bar{b}_{\lambda}\right\}_{\lambda \in \Lambda}$ be a basis for $B / \mathbf{m} B$ and map a free $A$-module $G=\bigoplus_{\lambda \in \Lambda} A$ to $B$. Then we have $B=$ $\operatorname{Im}(G)+\mathbf{m} \operatorname{Im}(G)$ and since $\mathbf{m}$ is nilpotent, Nakayama's Lemma gives $B=\operatorname{Im}(G)$ [17. If $K=\operatorname{ker}(G \rightarrow B)$, then we see that $K \otimes A / \mathbf{m}=0$ by flatness of $B$ over $A$, and so $K=0$ by another application of Nakayama's Lemma.

If $\mathbf{z}=z_{1}, \ldots, z_{d}$ are elements of a ring $S$, then we will use $(\mathbf{z})^{[t]}$ to denote the ideal $\left(z_{1}^{t}, \ldots, z_{d}^{t}\right) S$ for $t \geq 1$.

Theorem 3.3. Let $(R, \mathbf{m}, K) \rightarrow(S, \mathbf{n}, L)$ be a flat map of excellent rings with Gorenstein F-injective closed fiber. Assume that $K \rightarrow L$ is a separable field extension. Let $M=\lim R / I_{t}$ be an Artinian module which can be written as a direct limit of $\mathbf{m}$-primary ideals. Suppose that 0 is tightly closed in $M$, and let $\mathbf{z}=z_{1}, \ldots, z_{d} \in S$ be elements in $S$ which form an s.o.p. in $S / \mathbf{m} S$. Suppose that $c$ is a common $C S$ test element for $R$ and $S$. Then 0 is tightly closed in $M^{\prime}=\lim _{\longrightarrow} S /\left(I_{t}+(\mathbf{z})^{[t]}\right) S$.

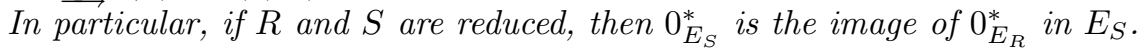

Proof. Let $b \in S$ map to the socle of $S /(\mathbf{m}+(\mathbf{z})) S$. For any element $u=\left\{u_{t}\right\} \in M$, suppose that for all $q$ there exists $t$ such that $c\left(u_{t}\left(z_{1} \cdots z_{d}\right)^{t-1} b\right)^{q} \in\left(I_{t}, z_{1}^{t}, \ldots, z_{d}^{t}\right)^{[q]}$. Then, since the fiber is CM, we have $c\left(u_{t} b\right)^{q} \in\left(I_{t}, \mathbf{z}\right)^{[q]}$. 
Given any choice of $u_{1}, \ldots, u_{n}$ generating the socle of $M$, the images of the set $\left\{u_{1} b, \ldots, u_{n} b\right\}$ generate the socle of $M^{\prime}$. If 0 is not tightly closed in $M^{\prime}$, then there is a socle element $v=\sum_{i=1}^{n} s_{i} u_{i} b$ in $0_{M^{\prime}}^{*}$, and the $s_{i}$ can be considered to be elements of $L$. Choose $u_{1}, \ldots, u_{n}$ so that such a sum can be written with the fewest possible $u_{i}$ 's. Let $h$ be this number and reorder so that $v=\sum_{i=1}^{h} s_{i} u_{i} b$. Suppose that the elements $s_{1}, \ldots, s_{h}$ (thought of as elements of $L$ ) are linearly dependent over $K$. Then without loss of generality, $s_{h}=r_{1} s_{1}+\cdots+r_{h-1} s_{h-1}$ and hence $v=\sum_{i=1}^{h-1} s_{i}\left(u_{i}+r_{i} u_{h}\right) b$, contradicting our choice of $h$. Also, $h \neq 1$, by Lemma 3.1.

We claim that for infinitely many $q$ there exist $t$ (depending on $q$ ) such that the set $\left\{\left(s_{1} b\right)^{q}, \ldots,\left(s_{h} b\right)^{q}\right\}$ is part of a free basis over $R / I_{t}^{[q]}$ for $S /\left(I_{t}, \mathbf{z}\right)^{[q]} S$. If not, then by Remark 3.2, for infinitely many $q$ there exist $r_{i q}$ not all in $\mathbf{m}$ such that $\sum_{i=1}^{h} r_{i q} s_{i}^{q} b^{q} \in \mathbf{m} S+(\mathbf{z})^{[q]} S$. Since $L$ is separable over $K$, each $\sum_{i=1}^{h} r_{i q} s_{i}^{q}$ is a unit (or else, modulo $\mathbf{n} S$, we have a relation on $s_{1}, \ldots, s_{h}$ over $K^{1 / q}$ ) and thus $b^{q} \in \mathbf{m} S+(\mathbf{z})^{[q]} S$, contradicting the $F$-injectivity of $S / \mathbf{m} S$. Now, if $c v^{q}=$ $\sum_{i=1}^{h}\left(c u_{i}^{q}\right)\left(s_{i} b\right)^{q}=0$ in $F^{e}\left(M^{\prime}\right)$ for infinitely many $q$, we must have $c u_{i}^{q}=0$ in $F^{e}(M)$ for infinitely many $q$ (by the assertion about free bases above). Thus $u_{i} \in$ $0_{M}^{*}$, a contradiction.

The last statement follows by taking $M=E_{R} / 0_{E_{R}}^{*}$. When $R$ is excellent and reduced, it is approximately Gorenstein, and $E_{R}$ may be written as a direct limit of the desired form. By Theorem 2.4, $E_{S}=\lim _{t} E_{R} \otimes_{R} S /(\mathbf{z})^{[t]}$.

Corollary 3.4. Let $(R, \mathbf{m})$ be an excellent reduced local ring. Then the tight closure of 0 in $E_{R}$ is independent of whether one computes it over $R$ or over $\widehat{R}$.

Proof. The closed fiber of $R \rightarrow \widehat{R}$ is a field. The residue fields are the same; so the extension of residue fields is separable. By Lemma $2.2, R$ and $\widehat{R}$ share a common CS test element. Thus Theorem 3.3 applies.

This shows that for $(R, \mathbf{m})$ excellent reduced, $\widetilde{\tau}(R)=\operatorname{Ann}_{R} 0_{E_{R}}^{*}=\widetilde{\tau}(\widehat{R}) \cap R$. Thus $\widetilde{\tau}(R) \widehat{R} \subseteq \widetilde{\tau}(\widehat{R})$. Below we show that equality does in fact hold.

By $(R, \underline{\mathbf{m}})$ we mean a semi-local ring with maximal ideals $\underline{\mathbf{m}}=\mathbf{m}_{1}, \ldots, \mathbf{m}_{t}$. By completion we mean completion at the Jacobson radical $\mathbf{m}_{1} \cap \cdots \cap \mathbf{m}_{t}$. This is then a product of complete local rings and $E_{R}=\bigoplus_{i=1}^{t} E_{R_{\mathbf{m}_{i}}}$. Note that when $(R, \underline{\mathbf{m}}) \rightarrow(S, \underline{\mathbf{n}})$ is flat with Gorenstein closed fibers and $0_{E_{S}}^{*}$ is extended from $0_{E_{R}}^{*}$, then $\widetilde{\tau}(R)=\widetilde{\tau}(S) \cap R$.

Remark 3.5. If $(S, \mathbf{m})$ is excellent, then the map $S \rightarrow \widehat{S}$ has geometrically regular fibers. This does not mean that for $Q \in \operatorname{Spec}(\widehat{S})$ and $P=Q \cap S$ the map of residue fields of $S_{P} \rightarrow \widehat{S}_{Q}$ is separable. However, if $Q$ is minimal over $P \widehat{S}$, then $\widehat{S}_{Q} / Q \widehat{S}_{Q}$ is a separable extension of $S_{P} / P S_{P}$.

The next theorem extends a result by Lyubeznik and Smith.

Theorem 3.6. Let $(S, \underline{\mathbf{n}})$ be a semi-local reduced excellent ring. Then

(1) $\widetilde{\tau}(S)$ localizes properly at any prime $Q \in \operatorname{Spec}(S)$, i.e., $\widetilde{\tau}(S)_{Q}=\widetilde{\tau}\left(S_{Q}\right)$.

(2) $\widetilde{\tau}(\widehat{S})=\widetilde{\tau}(S) \widehat{S}$. 
(3) Let $(R, \mathbf{m})$ be a semi-local reduced excellent ring such that $(R, \underline{\mathbf{m}}) \rightarrow(S, \underline{\mathbf{n}})$ is a flat semi-local map with Gorenstein F-injective closed fibers and such that if $\mathbf{n}$ is a maximal ideal of $S$ lying over $\mathbf{m}$, then $R / \mathbf{m} \rightarrow S / \mathbf{n}$ is separable. Assume that $R$ and $S$ have a common $C S$ test element. Then $\widetilde{\tau}(S)=\widetilde{\tau}(R) S$.

Proof. If the above statements do not hold, then take a counterexample with $S$ having the smallest possible dimension, and take it with the least of (1), (2), or (3) possible. Note that the statements hold for fields; so $\operatorname{dim} S>0$. Also, in each case we may immediately localize at a maximal ideal of $S$ (and its contraction in case $(3))$.

Suppose that the counterexample is to (1). Then by the induction hypothesis, for any chain of primes $Q \subsetneq P \subsetneq \mathbf{n}_{i}$ we have $\widetilde{\tau}\left(S_{Q}\right)=\widetilde{\tau}\left(S_{P}\right)_{Q}$. Consider $S \rightarrow \widehat{S}$. By [16], $\widetilde{\tau}(\widehat{S})$ localizes properly at any multiplicative system. Let $P \in \operatorname{Spec}(S)$ have dimension 1 (i.e., $\operatorname{dim} S / P=1$ ). Then $\widehat{S}_{P}$ is semi-local with maximal ideals precisely those primes of $\widehat{S}$ lying over $P \widehat{S}$ (which are necessarily minimal over $P \widehat{S}$ ). The induction hypothesis on (3) gives $\widetilde{\tau}\left(S_{P}\right) \widehat{S}_{P}=\widetilde{\tau}\left(\widehat{S}_{P}\right)$, and by [16], $\widetilde{\tau}\left(\widehat{S}_{P}\right)=$ $\widetilde{\tau}(\widehat{S})_{P}$. Thus

$$
\widetilde{\tau}\left(S_{P}\right)=\widetilde{\tau}\left(S_{P}\right) \widehat{S}_{P} \cap S_{P}=(\widetilde{\tau}(\widehat{S}))_{P} \cap S_{P}=(\widetilde{\tau}(\widehat{S}) \cap S)_{P}=\widetilde{\tau}(S)_{P}
$$

(The last equality follows from Corollary 3.4.) This contradicts $S$ being a counterexample.

Say the counterexample is to $(2)$. Then $\widetilde{\tau}(S) \widehat{S} \subseteq \widetilde{\tau}(\widehat{S})$, and we want to see that this is an equality. The associated primes of the extended ideal are primes of $\widehat{S}$ minimal among those lying over the associated primes of $\widetilde{\tau}(S)$. Say that $Q$ is such a prime (lying over $P$ ). Suppose first that $Q$ is not maximal. In this case we have $(\widetilde{\tau}(\widehat{S}))_{Q}=\widetilde{\tau}\left(\widehat{S}_{Q}\right)=\widetilde{\tau}\left(S_{P}\right) \widehat{S}_{Q}=\widetilde{\tau}(S)_{P} \widehat{S}_{Q}=(\widetilde{\tau}(S) \widehat{S})_{Q}$. The first equality follows since $\widetilde{\tau}$ localizes properly in complete rings, the second equality uses the induction hypothesis (3) (and Remark 3.5) in a map of rings of smaller dimension, and the third equality uses that $S$ has property (1).

We have reduced to the case that $\widetilde{\tau}(\widehat{S}) / \widetilde{\tau}(S) \widehat{S}$ is 0 -dimensional. So we may apply the following more general claim: if $A \subseteq E=E_{S}(L), I=(0: S A), J=\left(0:{ }_{\widehat{S}} A\right)$ and $\ell(J / I)<\infty$, then $J=I \widehat{S}$. To see this there is no loss of generality in modding out by $I$. Thus $A$ is an $S$-faithful submodule of $E$. If $J \neq 0$, then $\ell(J)<\infty$ implies that $\widehat{S}$, and therefore $S$, are depth 0 . Hence $J$ is extended from $R$, and $A$ is not faithful. Thus $J$ must be 0 .

Suppose now that we have a counterexample to (3). By Theorem $3.3,0_{E_{S}}^{*}$ is extended from $0_{E_{R}}^{*}$. The following lemma completes the proof.

Lemma 3.7. Let $(R, \mathbf{m}) \rightarrow(S, \mathbf{n})$ be a flat map of reduced local rings such that $S / \mathbf{m} S$ is Gorenstein and $0_{E_{S}}^{*}$ is extended. Assume also that $\widetilde{\tau}(\widehat{R})=\widetilde{\tau}(R) \widehat{R}$ and $\widetilde{\tau}(\widehat{S})=\widetilde{\tau}(S) \widehat{S}$. Then $\widetilde{\tau}(S)=\widetilde{\tau}(R) S$. If $0_{E_{S}}^{* f g}$ is extended, $\tau(\widehat{R})=\tau(R) \widehat{R}$, and $\tau(\widehat{S})=\tau(S) \widehat{S}$, then $\tau(S)=\tau(R) S$. 
Proof. Let $\mathbf{z}=z_{1}, \ldots, z_{t}$ be elements of $S$ which form an s.o.p. for $S / \mathbf{m} S$. Then we have

$$
\begin{aligned}
\operatorname{Hom}_{S}\left(S / \widetilde{\tau}(R) S, E_{S}\right) & =\operatorname{Hom}_{S}\left(R / \widetilde{\tau}(R) \otimes_{R} S, \underset{t}{\lim _{t}} E_{R} \otimes_{R} S /(\mathbf{z})^{[t]}\right) \\
& =\underset{t}{\lim } \operatorname{Hom}_{S}\left(R / \widetilde{\tau}(R) \otimes S /(\mathbf{z})^{[t]}, E_{R} \otimes S /(\mathbf{z})^{[t]}\right) \\
& =\underset{t}{\lim } \operatorname{Hom}_{R}\left(R / \widetilde{\tau}(R), E_{R}\right) \otimes S /(\mathbf{z})^{[t]} \\
& =\underset{t}{\lim } 0_{E_{R}}^{*} \otimes S /(\mathbf{z})^{[t]}=0_{E_{S}}^{*} .
\end{aligned}
$$

The last equality follows from the assumption that $0_{E_{S}}^{*}$ is extended. The second to last equality follows because $\widetilde{\tau}(\widehat{R})=\widetilde{\tau}(R) \widehat{R}$. The other equalities follow from the flatness of $S /(\mathbf{z})^{[t]} S$ over $R$ and standard facts about direct limits.

Also, since $\widetilde{\tau}(\widehat{S})=\widetilde{\tau}(S) \widehat{S}, 0_{E_{S}}^{*}=\operatorname{Hom}_{S}\left(S / \widetilde{\tau}(S), E_{S}\right)$. However, now by applying the exact functor $\operatorname{Hom}_{S}\left(-, E_{S}\right)$ to $0 \rightarrow C \rightarrow S / \widetilde{\tau}(R) S \rightarrow S / \widetilde{\tau}(S) \rightarrow 0$ we obtain that $\operatorname{Hom}_{S}\left(C, E_{S}\right)=0$. Hence $C=0$.

The last statement has the same proof.

Now that we have finished the proof that $\widetilde{\tau}$ extends properly, we may omit that hypothesis from Lemma 3.7.

Corollary 3.8. Let $(R, \mathbf{m}) \rightarrow(S, \mathbf{n})$ be a flat map of reduced local rings such that $S / \mathbf{m} S$ is Gorenstein and $0_{E_{S}}^{*}$ is extended. Then $\widetilde{\tau}(S)=\widetilde{\tau}(R) S$.

Proof. By Theorem 3.6(2), the additional condition needed in Lemma 3.7 is satisfied.

In the situation that $(R, \mathbf{m}) \rightarrow(S, \mathbf{n})$ is flat, we expect a common test element when the generic fibers are Gorenstein and $F$-rational. Below, we give a weaker condition which allows us to find a CS test element for $S$ which is almost as useful as a common test element.

Theorem 3.9. Let $(R, \mathbf{m}) \rightarrow(S, \mathbf{n})$ be a map of excellent reduced rings. Assume that $\mathbf{m} S$ is reduced in $S$ and set $U \subseteq S$ to be the complement of the minimal primes of $\mathbf{m} S$. Suppose that $R \rightarrow U^{-1} S$ is flat with $U^{-1} S / \mathbf{m} U^{-1} S$ separable over $R / \mathbf{m}$. Then there is an element $d \in U$ such that $d \widetilde{\tau}(R) \subseteq \widetilde{\tau}(S)$. In particular, $S$ has $C S$ test elements of the form $c d$, where $c$ is a $C S$ test element of $R$ and $d \in U$.

Proof. The map $R \rightarrow U^{-1} S$ is flat with Gorenstein closed fibers of dimension 0, and thus $E_{U^{-1} S}=E_{R} \otimes_{R} U^{-1} S$ by Theorem 2.4.

Let $Q$ be a minimal prime of $\mathbf{m} S$ and set $T=\widehat{S_{Q}}$. The separability hypothesis and Theorem 2.3 show that $\widehat{R}$ and $T$ have a common $C S$ test element, and thus by Theorem 3.3, $0_{T}^{*}$ is extended from $0_{\widehat{R}}^{*}$. Hence $0_{S_{Q}}^{*}=0_{T}^{*}$ is extended from $0_{\widehat{R}}^{*}=0_{R}^{*}$. By Theorem 3.6 and Corollary 3.8, $(\widetilde{\tau}(S))_{Q}=\widetilde{\tau}\left(S_{Q}\right)=\widetilde{\tau}(R) S_{Q}$ for each associated prime of $\mathbf{m} S$. Thus there is a $d \in U$ such that $d \widetilde{\tau}(R) S \subseteq \widetilde{\tau}(S)$.

We now state another version of Lemma 3.1.

Lemma 3.10. Let $(R, \mathbf{m}) \rightarrow(S, \mathbf{n})$ be a flat map of reduced local rings with $F$ rational closed fiber and also satisfying the conditions of Theorem 3.9. Suppose $\mathbf{z}=z_{1}, \ldots, z_{d}$ is an s.o.p. in $S / \mathbf{m} S$ and $b$ maps to a socle element in $S /(\mathbf{m}, \mathbf{z}) S$. If $M$ is an Artinian module and $u$ is not in $0_{M}^{*}$, then bu is not in $0_{M \otimes S /(\mathbf{z})}^{*}$. 
Proof. Let $M^{\prime}=M \otimes S /(\mathbf{z})$. Choose $c$ and $d$ as in Theorem 3.9. If the lemma is not true, then for all $q, c d(b u)^{q}=0$ in $F_{S}^{e}\left(M^{\prime}\right)$. Thus $d b^{q} \in 0: S \quad c u^{q}=$ $\left(0:_{R} c u^{q}\right) S+(\mathbf{z})^{[q]} S$, by flatness. Since $u \notin 0_{M}^{*},\left(0:_{R} c u^{q}\right) \subseteq \mathbf{m}$ for infinitely many $q$. Thus $d b^{q} \in\left((\mathbf{z})^{[q]}(S / \mathbf{m} S)\right)^{*}$, contradicting the $F$-rationality of $S / \mathbf{m} S$.

\section{$\S 4$. Extension of tight Closure By flat MAPS}

We are now in a position to consider the extension of tight closure under flat maps of local rings. In Theorem 4.1 we consider the case where the closed fiber is Gorenstein and either $F$-injective or $F$-rational. In Theorem 4.2 we weaken the hypothesis and assume only that the closed fiber is CM. As a trade-off, however, we must then assume that the closed fiber is either geometrically $F$-injective or geometrically $F$-rational. As an application, we extend the result (independently obtained by Enescu [7] and Hashimoto [8]) that the extension of an $F$-rational ring by a flat map with geometrically $F$-injective closed fiber is $F$-rational.

Theorem 4.1. Let $(R, \mathbf{m}, K) \rightarrow(S, \mathbf{n}, L)$ be a flat map of excellent rings with Gorenstein closed fiber. Assume that $K \rightarrow L$ is a separable field extension. Let $\mathbf{z}=z_{1}, \ldots, z_{d} \in S$ be elements in $S$ which form an s.o.p. in $S / \mathbf{m} S$. Suppose that

(1) c is a common CS test element for $R$ and $S$, and $S / \mathbf{m} S$ is F-injective; or

(2) $S / \mathbf{m} S$ is F-rational and $K \rightarrow S / \mathbf{m} S$ is separable.

Let $M$ be an R-module with DCC, and set $M^{\prime}=M \otimes_{R} \lim _{t} S /(\mathbf{z})^{[t]} S$. Then $0_{M^{\prime}}^{*}$ (respectively $0_{M^{\prime}}^{* f g}$ ) is extended from $0_{M}^{*}$ (respectively $0_{M}^{* f g}$ ). In particular, if $I$ is a tightly closed $\mathbf{m}$-primary ideal of $R$, then $\left(I+(\mathbf{z})^{[t]}\right) S$ is a tightly closed ideal of $S$ for all $t$.

Proof. We may start by modding out by $0_{M}^{*}$ (respectively $0_{M}^{* f g}$ ) to assume that 0 is (finitistically) tightly closed in $M$. Thus we wish to show that 0 is also (finitistically) tightly closed in $M^{\prime}$. Note that since $S / \mathbf{m} S$ is Gorenstein, z forms a regular sequence on $M \otimes_{R} S$. Hence, the maps $M \otimes_{R} S /(\mathbf{z})^{[t]} S \rightarrow M \otimes_{R} S /(\mathbf{z})^{[t+1]} S$ are injective. So any socle element in $M^{\prime}$ is already present in $M \otimes S /(\mathbf{z}) S$.

The initial setup here is as in Theorem 3.3. Let $b \in S$ map to the socle of $S /(\mathbf{m}+(\mathbf{z})) S$. Given any choice of $u_{1}, \ldots, u_{n}$ generating the socle of $M$, the set $\left\{u_{1} b, \ldots, u_{n} b\right\}$ generates the socle of $M^{\prime}$ (the closed fiber is Gorenstein). If 0 is not tightly closed in $M^{\prime}$, then there is a socle element $v=\sum_{i=1}^{n} s_{i} u_{i} b \in 0_{M^{\prime}}^{*}$, and the $s_{i}$ can be considered to be elements of $L$. Choose $u_{1}, \ldots, u_{n}$ so that such a sum can be written with the fewest possible $u_{i}^{\prime} s$. Let $h$ be this number and reorder so that $v=\sum_{i=1}^{h} s_{i} u_{i} b$. Suppose that the elements $s_{1}, \ldots, s_{h}$ (thought of as elements of $L$ ) are linearly dependent over $K$. Then without loss of generality, $s_{h}=r_{1} s_{1}+\cdots+r_{h-1} s_{h-1}$ and hence $v=\sum_{i=1}^{h-1} s_{i}\left(u_{i}+r_{i} u_{h}\right) b$, contradicting our choice of $h$. Also, $h \neq 1$ by Lemma 3.1.

In both cases we can choose $c$ and $d$ as in Theorem 3.9 (where in case (1) we may assume that $d=1$ ).

Since $v \in 0_{M^{\prime}}^{*}$ we have that $c d v^{q}=0$ in $F_{S}^{e}\left(M^{\prime}\right)$. Using the definition of the direct limit and that $\mathbf{z}$ is a regular sequence on $M \otimes_{R} S$, we get $c d v^{q}=0$ in $F_{S}^{e}\left(S /(\mathbf{z}) S \otimes_{R} M\right)$.

We have $d b^{q} \in\left(c\left(u_{1}, \ldots, u_{h-1}\right)^{[q]}:_{S} c u_{h}^{q}\right)$ where the elements defining the colon ideal belong to $F_{S}^{e}\left(S /(\mathbf{z}) S \otimes_{R} M\right)$. As we noted earlier, $R \rightarrow S /\left(\mathbf{z}^{[q]}\right) S$ is flat, and so 
$d b^{q} \subseteq\left(\left(c\left(u_{1}, \ldots, u_{h-1}\right)^{[q]}\right):_{R} c u_{h}^{q}\right) S+(\mathbf{z})^{[q]} S$ for all $q$, where the elements defining the colon ideal belong to $F_{R}^{e}(M)$.

If this colon ideal is contained in $\mathbf{m}$ for $q \gg 0$, then we get a contradiction (see the cases listed below). So we may assume that for large enough $q_{1}, c u_{h}^{q_{1}}=$ $r_{h, 1} c u_{1}^{q_{1}}+\cdots+r_{h, h-1} c u_{h-1}^{q_{1}}$ in $F_{R}^{e_{1}}(M)$. Then $c d v^{q_{1}}=\sum_{i=1}^{h-1} c d\left(s_{i}^{q_{1}}+r_{h, i} s_{h}^{q_{1}}\right) u_{i}^{q_{1}} b^{q_{1}}$. Each $s_{i}^{q_{1}}+r_{h, i} s_{h}^{q_{1}}$ must be a unit, since $L$ is separable over $K$. Thus we have $d b^{q} \in c\left(u_{1}, \ldots, u_{h-2}\right)^{[q]}:_{S} c u_{h-1}^{q} \subseteq\left(\left(c\left(u_{1}, \ldots, u_{h-2}\right)^{[q]}\right):_{R} c u_{h-1}^{q}\right) S+(\mathbf{z})^{[q]} S$ for all $q \gg 0$. We obtain a contradiction (see the cases listed below) unless for all $q_{2} \gg 0, c u_{h-1}^{q_{2}}=c r_{h-1,1} u_{1}^{q_{2}}+\cdots+c r_{h-1, h-2} u_{h-2}^{q_{2}}$ in $F_{R}^{e_{2}}(M)$. Then $c d v^{q_{2}}=$ $\sum_{i=1}^{h-2}\left(s_{i}^{q_{2}}+r_{h, i}^{q_{2} / q_{1}} s_{h}^{q_{2}}+r_{h-1, i} s_{h-1}^{q_{2}}\right) u_{i}^{q_{2}} b^{q_{2}}$. Again, each coefficient must be a unit by separability. Continuing in this manner we get a unit $\alpha \in S$ and $q$ such that in the respective cases:

(1) $v^{q}=\alpha u_{1}^{q} b^{q}=0$ in $F_{S}^{e}\left(M^{\prime}\right)$, implying that $b^{q} \in(\mathbf{z})^{[q]}(S / \mathbf{m} S)$, contradicting the $F$-injectivity of $S / \mathbf{m} S$ (look, for example, at the proof of Lemma 3.1);

(2) $v^{q}=\alpha u_{1}^{q} b^{q} \in 0_{F_{S}^{e}\left(M^{\prime}\right)}^{*}$, contradicting Lemma 3.10.

The case of the finitistic tight closure of 0 is similar and we will leave it as an exercise to the reader.

Now we would like to treat the case when the closed fiber is Cohen-Macaulay, and not necessarily Gorenstein.

Theorem 4.2. Let $(R, \mathbf{m}, K) \rightarrow(S, \mathbf{n}, L)$ be a flat map of excellent rings with $C M$ closed fiber. Let $I \subseteq R$ be $\mathbf{m}$-primary and tightly closed. Let $\mathbf{z}=z_{1}, \ldots, z_{d}$ be elements whose image in $S / \mathbf{m} S$ is an s.o.p. Suppose that either

(1) the element $c \in R^{\circ}$ is a common test element for $R$ and $S$, and that $S / \mathbf{m} S$ is geometrically $F$-injective over $K$, or

(2) $S / \mathbf{m} S$ is geometrically $F$-rational over $K$.

Then $(I, \mathbf{z}) S$ is tightly closed.

Proof. Let $\left\{b_{1}, \ldots, b_{m}\right\}$ map to generators of the socle of $S /(\mathbf{m}, \mathbf{z}) S$. If $(I, \mathbf{z}) S$ is not tightly closed, then for any choice of generators of the socle of $R / I$, say $\left\{u_{1}, \ldots, u_{n}\right\}$, there exists a socle element $w=\sum_{i, j} \lambda_{i j} u_{j} b_{i}$ in $((I, \mathbf{z}) S)^{*}$. Choose $\left\{u_{1}, \ldots, u_{n}\right\}$ and $w$ to minimize the number of nonzero $\lambda_{i j}$. We first observe that $\left\{\lambda_{i j} b_{i}\right\}_{i, j}$ is part of a minimal basis for $S / I S$ over $R / I$. Otherwise, by Remark 3.2 there exist $k_{i j} \in K$ such that $\sum_{i, j} k_{i j} \lambda_{i j} b_{i}=0$ in $S /(\mathbf{m}, \mathbf{z}) S$. This would allow us to choose $\left\{u_{j}^{\prime}\right\}$ to get a smaller number of nonzero $\lambda_{i j}$.

We claim that in fact $\left\{\lambda_{i j}^{q} b_{i}^{q}\right\}_{i, j}$ is part of a free $R / I^{[q]}$-basis for $S /(I, \mathbf{z})^{[q]}$ for every $q$. If not, for some $q$ there exist $k_{q i j}=k_{i j} \in K$ such that $\sum_{i, j} k_{i j} \lambda_{i j}^{q} b_{i}^{q}=0$ in $S /\left(\mathbf{m},(\mathbf{z})^{[q]}\right) S$. Let $K^{\prime}=K\left[k_{i j}^{1 / q}\right]$ and then the element $\left(\sum_{i, j} k_{i j}^{1 / q} \lambda_{i j} b_{i}\right)^{q}=0$ in $S /\left(\mathbf{m},(\mathbf{z})^{[q]}\right) S \otimes K^{\prime}$. However, $S / \mathbf{m} S \otimes K^{\prime}$ is $F$-injective; so $\sum_{i, j} k_{i j}^{1 / q} \lambda_{i j} b_{i}=0$ in $S /(\mathbf{m}, \mathbf{z}) S \otimes K^{\prime}$, a contradiction.

In case $(1), c w^{q}=\sum_{i, j}\left(c u_{j}^{q}\right) \lambda_{i j}^{q} b_{i}^{q} \in(I, \mathbf{z})^{[q]}$ then implies that $c u_{j}^{q} \in I^{[q]} R$, or $u_{j} \in I^{*}=I$, a contradiction.

In case (2), choose $c \in R^{\circ}$ and $d \in S^{\circ}$ as in Theorem 3.9. We claim that the set $\left\{d \lambda_{i j}^{q} b_{i}^{q}\right\}_{i, j}$ is part of a free $R / I^{[q]}$-basis for $S /(I, \mathbf{z})^{[q]}$ for infinitely many $q$. Otherwise, as in the analysis above we get $d\left(\sum_{i, j} k_{q i j}^{1 / q} \lambda_{i j} b_{i}\right)^{q} \in(\mathbf{z})^{[q]} S / \mathbf{m} S \otimes K^{\infty}$. Each $w_{q}=\sum_{i, j} k_{q i j}^{1 / q} \lambda_{i j} b_{i}$ is a socle element in $S /(\mathbf{m}, \mathbf{z}) \otimes K^{\infty}$, and the socle is 
finite dimensional as a $K^{\infty}$ vector space. Let $V_{q}$ be the $K^{\infty}$-subspace of the socle generated by $\left\{w_{q^{\prime}}\right\}_{q^{\prime}} \geq q$. Then by $D C C$ there exists $q_{0}$ such that $V_{q}=V_{q_{0}}$ for all $q \geq q_{0}$. Let $K^{\prime}=K\left[k_{q_{0} i j}^{1 / q_{0}}\right]$ (this is a module-finite extension of $K$ ). Then $d w_{q_{0}}^{q} \in d\left(w_{q^{\prime}} \mid q^{\prime} \geq q_{0}\right)^{[q]} \cap S / \mathbf{m} S \otimes K^{\prime} \subseteq(\mathbf{z})^{[q]} S / \mathbf{m} S \otimes K^{\prime}$. However, this shows that a socle element is in the tight closure of $S /(\mathbf{m}, \mathbf{z}) \otimes K^{\prime}$, contradicting the $F$-rationality of $S / \mathbf{m} S \otimes K^{\prime}$.

We can now improve Enescu's and Hashimoto's theorem on extension of Frational rings by flat maps with sufficiently nice generic and closed fibers.

Theorem 4.3. Let $(R, \mathbf{m}, K) \rightarrow(S, \mathbf{n}, L)$ be a flat map of excellent rings. Assume that $R$ is F-rational. Suppose that either

(1) $c \in R^{\circ}$ is a common parameter test element for $R$ and $S$ and $S / \mathbf{m} S$ is geometrically $F$-injective, or

(2) $S / \mathbf{m} S$ is geometrically $F$-rational.

Then $S$ is F-rational.

Proof. If $R$ and $S$ have a common parameter test element, then case (1) of Theorem 4.2 applies with $I \subseteq R$ a parameter ideal (this case is known already by the results of Enescu [7] and of Hashimoto [8], under some finiteness conditions). Otherwise case (2) applies.

The next proposition generalizes 9], Theorem 7.36, where the closed fiber is assumed to be regular.

Proposition 4.4. Let $(R, \mathbf{m}, K) \rightarrow(S, \mathbf{n}, L)$ be a flat map of reduced excellent rings with Gorenstein closed fiber such that $R$ is complete and either

(1) $S / \mathbf{m} S$ is F-injective, $K \subset L$ is separable and $R$ and $S$ have a common test element;

(2) $S / \mathbf{m} S$ is F-rational and $K \rightarrow S / \mathbf{m} S$ is separable;

(3) the element $c \in R^{\circ}$ is a common test element for $R$ and $S$, and $S / \mathbf{m} S$ is geometrically $F$-injective, or

(4) $S / \mathbf{m} S$ is geometrically $F$-rational over $K$.

Then $\tau(R) S=\tau(S)$.

Proof. Both $R$ and $S$ are approximately Gorenstein. If $I_{t}$ is a sequence of irreducible $\mathbf{m}$-primary ideals cofinal with powers of $\mathbf{m}$, then $\tau(R)=\bigcap I_{t}: I_{t}^{*}$. Let $\mathbf{z}=$ $z_{1}, \ldots, z_{d} \in S$ be an s.o.p. for $S / \mathbf{m} S$. Now, $J_{t}=\left(I_{t},(\mathbf{z})^{[t]}\right) S$ is a sequence of irreducible ideals in $S$ (because $S / \mathbf{m} S$ is Gorenstein). By Theorems 4.1 and 4.2, $J_{t}^{*}=\left(I_{t}^{*},(\mathbf{z})^{[t]}\right) S$. Because of the flatness of the original map, we get that $J_{t}: J_{t}^{*}=$ $\left(I_{t}, \mathbf{z}^{[t]}\right) S: S\left(I_{t}^{*}, \mathbf{z}^{[t]}\right) S=\left(I_{t}^{*}:_{R} I_{t}\right) S+(\mathbf{z})^{[t]} S$. Hence,

$$
\begin{aligned}
\tau(S)=\bigcap_{t}\left(J_{t}: S J_{t}^{*}\right)=\bigcap_{t}\left(I_{t}:_{R} I_{t}^{*}\right) S+(\mathbf{z})^{[t]} \\
\quad=\bigcap_{t^{\prime}}\left(\left(\bigcap_{t}\left(I_{t}:_{R} I_{t}^{*}\right) S\right)+(\mathbf{z})^{\left[t^{\prime}\right]}\right)=\bigcap_{t^{\prime}}\left(\tau(R) S+(\mathbf{z})^{\left[t^{\prime}\right]}\right)=\tau(R) S
\end{aligned}
$$

(in the next to last equality we use that $R$ is complete and hence $S$ is $\cap$-flat over $R)$. 
Remark 4.5. Recently, Bravo and Smith have studied the behavior of test ideals under smooth and étale base change using different methods than ours ([5]). Their results are stated under the assumption that the test ideal localizes or that the finitistic tight closure of zero equals the tight closure of zero in the injective hull of a reduced local excellent ring. These are hard open questions that are likely to be true under very mild conditions. Our results give some more general conditions under which the test ideal extends naturally via flat base change. The assumption that $R$ is complete is not very restrictive. Comparing to the results of Bravo and Smith, if the test ideal localizes, it also extends well under completion. A proof of this can be given, following closely the proof of Theorem 3.6 (one needs to use Theorem 4.1 instead of Theorem 3.3 whenever it is needed). Also, if the finitistic tight closure of 0 equals the tight closure of 0 in the injective hull, then the test ideal equals the CS test ideal which behaves well under completion (cf. Theorem 3.6).

\section{§5. Strong test ideals AND APplications}

An ideal $T \subseteq R$ is said to be a strong test ideal for $R$ if $T I^{*}=T I$ for all ideals $I \subseteq R$ (see 13]). Vraciu has shown that in a complete reduced ring $(R, \mathbf{m}, k)$, if $T$ is the annihilator of any finitistically tightly closed submodule of $E_{R}(k)$, then $T$ is a strong test ideal [21], Theorem 3.2. As a corollary we now obtain

Theorem 5.1. Let $(R, \mathbf{m}, K)$ be an excellent reduced ring. Then $\widetilde{\tau}(R)$ is a strong test ideal for $R$.

Proof. This follows immediately from the faithful flatness of $R \rightarrow \widehat{R}$ and from the fact that $\widetilde{\tau}(\widehat{R})=\widetilde{\tau}(R) \widehat{R}$.

We would like to show that $\widetilde{\tau}(R)$ is a strong test ideal in the CS sense, i.e., if $M=\varliminf_{t} R / I_{t}$ is Artinian, and $w=\left\{w_{t}\right\} \in 0_{M}^{*}$, then for some $t, \widetilde{\tau}(R) w_{t} \in \widetilde{\tau}(R) I_{t}$. We have not been able to prove this; however, we can prove a slightly weaker result which will suffice for our main application.

Theorem 5.2. Let $(R, \mathbf{m}, K)$ be a reduced excellent ring. Suppose that $M=$ $\lim _{t} R / I_{t}$, where each $I_{t}$ is $\mathbf{m}$-primary. Let $w=\left\{w_{t}\right\} \in 0_{M}^{*}$. Then for all $N>0$ there exists $t$ such that $\widetilde{\tau}(R) w_{t} \in\left(\widetilde{\tau}(R)+\mathbf{m}^{N}\right) I_{t}$.

Proof. We give a proof here that is modeled after Vraciu's proof of [21], Theorem 3.2. As in the proof of Theorem 5.1, it suffices to show that the statement holds when $R$ is complete. Let $\widetilde{\tau}=\widetilde{\tau}(R)$.

Fix $N>0$. Let $M_{1}=\lim _{\longrightarrow} R /\left(\widetilde{\tau}+\mathbf{m}^{N}\right) I_{t}$. This makes sense because for any ideal $A$ and any map $R / I \rightarrow R / J$ there is a clear induced map $R / A I \rightarrow R / A J$. The module $M_{1}$ is supported only at $\mathbf{m}$. Let $w^{\prime}=\left\{w_{t}^{\prime}\right\} \in M_{1}$ map to $w$ in $M$.

We observe that $0:_{M_{1}} \tilde{\tau}=\lim _{t}\left(\left(\left(\widetilde{\tau}+\mathbf{m}^{N}\right) I_{t}\right): \widetilde{\tau}\right) /\left(\widetilde{\tau}+\mathbf{m}^{N}\right) I_{t}$ and clearly $I_{t} \subseteq\left(\left(\left(\widetilde{\tau}+\mathbf{m}^{N}\right) I_{t}\right): \widetilde{\tau}\right)$ and so $w^{\prime} \in\left(0:_{M_{1}} \widetilde{\tau}\right)_{M_{1}}^{*}$.

Since $M_{1}$ is supported only at $\mathbf{m}$, we get an embedding $M_{1} \rightarrow G$ where $G$ is a (possibly infinite) direct sum of $E_{R}(k)$ 's. Then $0:_{M_{1}} \widetilde{\tau}=M_{1} \cap\left(0:_{G} \widetilde{\tau}\right)$. Since $(0: G \widetilde{\tau})$ is tightly closed, so is $0:_{M_{1}} \widetilde{\tau}$. By the remark following Proposition 2.1, $\widetilde{\tau}$ kills tight closure in all the modules involved, and hence there exists $t$ such that $\widetilde{\tau} w_{t} \in\left(\widetilde{\tau}+\mathbf{m}^{N}\right) I_{t}$ 
The next theorem shows that tight closure in the injective hull may be shown to extend without the need for a common CS test element as long as the extension of residue fields is separable. Because we will make use of a result from [3] (see Theorem 5.4 below) which is proved for rings whose completions are domains, we will restrict our statement to that case. We believe, however, that Theorem 5.4 is still true for reduced equidimensional rings, so that Theorem 5.3 should also hold under these more general assumptions.

Theorem 5.3. Let $(R, \mathbf{m}, K) \rightarrow(S, \mathbf{n}, L)$ be a flat map of excellent domains both of whose completions are domains. Assume that $S / \mathbf{m} S$ is Gorenstein and $F$-rational and $K \rightarrow L$ is separable. Then $0_{E_{S}}^{*}$ is extended from $0_{E_{R}}^{*}$, and $\widetilde{\tau}(S)=\widetilde{\tau}(R) S$.

Also, $0_{E_{S}}^{* f g}$ is extended from $0_{E_{R}}^{* f g}$ and hence $R$ and $S$ have a common test element (we cannot make the assertion that $\tau(S)=\tau(R) S$ from this).

Proof. Choose $\mathbf{z}=z_{1}, \ldots, z_{d}$ an s.o.p. for $S / \mathbf{m} S$ and let $b$ be the socle element in $S /(\mathbf{m}+\mathbf{z})$. Let $u_{1}, \ldots, u_{h}$ generate the socle modulo $0_{E_{R}}^{*}$ where we write $E_{R}(k)$ as $\lim _{t} R / I_{t}$.

Suppose now that $0_{E_{S}}^{*}$ is not extended. Then there is an element $x=\left(\sum s_{j} u_{j}\right) b$ in $0_{E_{S}}^{*}$, i.e., there is a $d \in S^{\circ}$ such that for all $q$ there exists $t$ such that $d\left(\sum s_{j}^{q} u_{j, t}^{q}\right) b^{q}$ $\in\left(I_{t}, \mathbf{z}\right)^{[q]} S$.

We can choose $x$ and $u_{1}, \ldots, u_{h}$ so that the number of nonzero terms in the expression of $x$ is minimal. As in the proof of Theorem 4.1, it follows that the coefficients $s_{j}$ are linearly independent over $K$ when regarded as elements of $L$.

We can also arrange that for some $w, u_{w+1}, \ldots, u_{h} \in\left(u_{1}, \ldots, u_{w}\right)_{E_{R}}^{*}$, but $u_{w} \notin$ $\left(u_{1}, \ldots, u_{w-1}\right)_{E_{R}}^{*}$. Also, suppose that $\widetilde{\tau}(R)=\left(c_{1}, \ldots, c_{l}\right)$ minimally.

As in the proof of Theorem 4.1, we can easily show that $b u_{w} \notin\left(u_{1}, \ldots, u_{w-1}\right)_{E_{S}}^{*}$ in $E_{S}$.

Fix $N>0$. According to Theorem 5.2, for every $q$ we can find $t$ such that

$$
\widetilde{\tau}(R)\left(u_{t, w+1}, \ldots, u_{t, h}\right)^{[q]} \subseteq\left(\widetilde{\tau}(R)+\mathbf{m}^{N}\right)\left(u_{t, 1}, \ldots, u_{t, w}, I_{t}\right)^{[q]} .
$$

Multiply now by $c_{1}$ and use Theorem 5.2 to get for each $q$, an integer $t$ such that (omitting the $t$ indices on the left)

$$
d\left(\sum_{j=1}^{w}\left(c_{1} s_{j}^{q}+\sum_{v=w+1}^{h} s_{v}^{q} r_{j, v, q}\right) u_{j}^{q}\right) b^{q} \in\left(I_{t}, \mathbf{z}\right)^{[q]} S .
$$

Here the $r_{j, v, q}$ 's are in $\widetilde{\tau}(R)+\mathbf{m}^{N}$. We then have

$$
\begin{aligned}
d\left(c_{1} s_{w}^{q}+\sum_{v=w+1}^{h} s_{v}^{q} r_{w, v, q}\right) b^{q} & \in\left(\left(u_{1}, \ldots, u_{w-1}, I_{t}, \mathbf{z}\right)^{[q]}:_{S} u_{w}^{q}\right) \\
& =\left(\left(u_{1}, \ldots, u_{w-1}, I_{t}\right)^{[q]}:_{R} u_{w}^{q}\right) S+(\mathbf{z})^{[q]} S
\end{aligned}
$$

and, since $u_{w} \notin\left(u_{1}, \ldots, u_{w-1}\right)_{E_{R}}^{*}$, by Theorem 5.4 below there is a $q_{0}$ such that

$$
\left(u_{1}, \ldots, u_{w-1}, I_{t}\right)^{[q]}:_{R} u_{w}^{q} \subseteq \mathbf{m}^{\left[q / q_{0}\right]} .
$$

Note that $b^{q_{0}} \notin\left(\left(\mathbf{m}+(\mathbf{z})^{\left[q_{0}\right]}\right) S\right)^{*}$ (or else $\left.b \in((\mathbf{z}) S / \mathbf{m} S)^{*}\right)$. So

$$
\left(c_{1}\left(s_{w}^{q}+\cdots+r_{h, q}^{\prime} s_{h}^{q}\right)+c_{q}\right) \in\left(\mathbf{m}+(\mathbf{z})^{\left[q_{0}\right]}\right) S:_{S} d\left(b^{q_{0}}\right)^{q / q_{0}} \subseteq \mathbf{n}^{\left[q / q_{1}\right]}
$$


for some $q_{1} \geq q_{0}$. The element $c_{q}$ is in $\left(c_{2}, \ldots, c_{l}\right)+\mathbf{m}^{N}$. By separability of $L$ over $K$, the coefficient of $c_{1}$ must be a unit for all $q$. Thus, by applying the Krull intersection theorem we see that $c_{1}$ is in

$$
\begin{aligned}
\left(\bigcap_{q}\left(\left(c_{2}, \ldots, c_{h}\right)+\mathbf{m}^{N}+\mathbf{n}^{\left[q / q_{1}\right]}\right)\right) \cap R \subseteq\left(\left(c_{2}, \ldots, c_{h}\right)\right. & \left.+\mathbf{m}^{N}\right) S \cap R \\
& =\left(\left(c_{2}, \ldots, c_{h}\right)+\mathbf{m}^{N}\right) R .
\end{aligned}
$$

This argument is valid for all $N>0$. So another application of the Krull intersection theorem shows that $c_{1} \in\left(c_{2}, \ldots, c_{l}\right)$, a contradiction. By Corollary 3.8, $\widetilde{\tau}(S)=$ $\widetilde{\tau}(R) S$.

The proof for the finitistic tight closure is exactly the same, although we may use Vraciu's original result, since we may immediately fix a value of $t$.

The next Theorem appears as [3], Proposition 2.4. We have altered the statement slightly to include the element $d \in R^{\circ}$, but the proof is essentially the same.

Theorem 5.4. Let $(R, \mathbf{m})$ be an excellent local domain such that its completion is a domain. Let $M=\varliminf_{t} R / I_{t}$ be a direct limit system. Fix $u \notin 0_{M}^{*}$ and $d \in R^{\circ}$. Then there exists $q_{0}$ such that $J_{q}=\bigcup_{q}\left(I_{t}^{[q]}: d u_{t}^{q}\right) \subseteq \mathbf{m}^{\left[q / q_{0}\right]}$ for all $q \gg 0$ (where $\left\{u_{t}\right\}$ represents $u \in M$ and $\left.u_{t} \mapsto u_{t+1}\right)$. In particular, if $I \subseteq R$ we may take $M=R / I$ where the limit system consists of equalities. Then $u \notin I^{*}$ implies that $\left(I^{[q]}: d u^{q}\right) \subseteq \mathbf{m}^{\left[q / q_{0}\right]}$.

Remark 5.5. As mentioned above, Theorem 5.3 should be true whenever $R$ and $S$ are reduced and equidimensional (and excellent). The proof given above does give the result when the completion of $R$ is a domain, the closed fiber of the map is regular, $S$ is reduced (not necessarily equidimensional) and $K \subseteq L$ is separable. This is because we may pick $\mathbf{z}$ to be a regular system of parameters for $S / \mathbf{m} S$ and take $b=1$. When $b=1$, using that there is $q_{0}$ such that $\left(u_{1}, \ldots, u_{w-1}\right)^{[q]}:_{R}$ $d u_{w}^{q}+I_{t}^{[q]} \subseteq \mathbf{m}^{\left[q / q_{0}\right]}$, one has that $c_{1}\left(s_{w}^{q}+\cdots+r_{h, q}^{\prime} s_{h}^{q}\right)+c_{q} \in\left(\mathbf{m}+(\mathbf{z})^{\left[q_{0}\right]}\right)^{\left[q / q_{0}\right]}$, which is clearly in larger and larger powers of $\mathbf{n}$ as $q$ increases. The rest of the argument then follows.

We can now give an improved version of Theorem 3.9.

Theorem 5.6. Let $(R, \mathbf{m}) \rightarrow(S, \mathbf{n})$ be a map of excellent reduced rings. Assume that $\widehat{R}$ is a domain. Let $Q \in \operatorname{Spec}(S)$ be such that $\mathbf{m} S \subseteq Q, R \rightarrow S_{Q}$ is flat, $\mathbf{m} S_{Q}$ is reduced, and $R / \mathbf{m} \rightarrow S_{Q} / Q S_{Q}$ is separable. Assume that either

(1) $\widehat{S_{Q}}$ is a domain and $\widehat{S_{Q}} / \mathbf{m} \widehat{S_{Q}}$ is Gorenstein and F-rational, or

(2) $S_{Q} / \mathbf{m} S_{Q}$ is regular.

Then there is an element $d \in S-Q$ such that $d \widetilde{\tau}(R) \subseteq \widetilde{\tau}(S)$.

Proof. Given Theorem 5.3 (or Remark 5.5), the proof is identical to that of Theorem 3.9 .

We will end with several more consequences of the ideas used in the proof of Theorem 3.3. The first one addresses the notion of Hilbert-Kunz multiplicities. For an $\mathbf{m}$-primary ideal $I$ in a local $\operatorname{ring}(R, \mathbf{m}, K)$ of Krull dimension $d$, the HilbertKunz multiplicity of $I$ is defined as $e_{H K}(I, R)=\lim _{q \rightarrow \infty} l_{R}\left(R / I^{[q]}\right) / q^{d}$. If $I=\mathbf{m}$, we will just write $e_{H K}(R)$. 
Proposition 5.7. Let $(R, \mathbf{m}, K) \rightarrow(S, \mathbf{n}, L)$ be a flat local map with a CohenMacaulay closed fiber. Then for every $\mathbf{m}$-primary ideal I and for every s.o.p. $\mathbf{z}$ of the closed fiber $S / \mathbf{m} S$,

$$
e_{H K}(I, R) e_{H K}(S / \mathbf{m} S)=e_{H K}(I S+\mathbf{z} S, S) .
$$

Proof. As before, $R / I R \rightarrow S /(I S+\mathbf{z} S)$ is flat. Apply Remark 3.2 and deduce that in fact $S /(I S+\mathbf{z}) S$ is free over $R / I$ of rank equal to the rank of $S /(\mathbf{m} S+\mathbf{z} S)$ over $K$. We can apply this for $I^{[q]}$ and $z^{[q]}$. Denote by $d$ and $e$ the Krull dimension of $R$ and $S / \mathbf{m} S$, respectively. In fact, it follows that

$$
l_{S}\left(S /\left(I^{[q]}, \mathbf{z}^{[q]}\right) S\right)=l_{R}\left(R / I^{[q]}\right) l_{S}\left(S /\left(\mathbf{m} S+\mathbf{z}^{[q]}\right) S\right)
$$

(one can see this by applying [6], Exercise 1.2.25). Hence,

$$
\left(1 / q^{(d+e)}\right) l_{S}\left(S /\left(I^{[q]}+\mathbf{z}^{[q]}\right) S\right)=\left(1 / q^{d}\right) l_{R}\left(R / I^{[q]}\right)\left(1 / q^{e}\right) l_{S / \mathbf{m} S}\left(S /\left(\mathbf{m}+\mathbf{z}^{[q]}\right) S\right) .
$$

Taking the appropriate limits we get the stated identity.

It might be helpful to note the following:

Corollary 5.8 (Kunz). In the situation stated above, $e_{H K}(S) \leq e(S / \mathbf{m} S) e_{H K}(R)$. Here, $e(S / \mathbf{m} S)$ stands for the multiplicity of $S / \mathbf{m} S$.

Proof. Take $I=m$ and use the fact that $e_{H K}(S) \leq e_{H K}(m S+z S)$ and that $e_{H K}(S / m S) \leq e(S / m S)$.

This Corollary has been obtained earlier by Kunz in 1976 with a different proof.

Lastly we would like to show how this circle of ideas may be applied to the theory of test exponents, a notion introduced recently by Hochster and Huneke [12. The notion is intimately connected to the localization problem in tight closure theory.

First, we would like to remind the reader of the definition of a test exponent. Let $R$ be a reduced Noetherian ring of positive characteristic, $c$ a fixed test element for $R$, and $I$ an ideal of $R$. We say that $Q$ is a test exponent for $c, I$ if whenever $c u^{q} \in I^{[q]}$ and $q \geq Q$, then $u \in I^{*}$. The minimal test exponent for $c, I$ is denoted by $\exp (c, I, R)$, if it exists.

Proposition 5.9. Let $(R, \mathbf{m}, K) \rightarrow(S, \mathbf{n}, L)$ be a flat local map with Gorenstein $F$-injective closed fiber. Assume that $K \subset L$ is separable. Let $c$ be a common completely stable test element of $R$ and $S$. If a test exponent exists for $c^{2}, I, R$, then a test exponent exists for $c, I S+\mathbf{z} S, S$. Moreover, $\exp \left(c^{2}, I, R\right) \geq \exp (c, I S+\mathbf{z} S, S)$. Here, $\mathbf{z}$ stands for a system of parameters of the closed fiber $S / \mathbf{m} S$.

Proof. Denote by $Q$ the test exponent of $c^{2}$ with respect to $I$ and $R$. We will freely use the notation of Theorem 3.3 for the case $M=R / I$. We want to show that if $c v^{q} \in I^{[q]} S+(\mathbf{z})^{q} S$ (let us denote this relation by (\#)) for some $q \geq Q$, then $v \in(I S+\mathbf{z} S)^{*}$. We know that $(I S+\mathbf{z} S)^{*}=\left(I^{*} S+\mathbf{z}\right) S$. Fix $q \geq Q$ and denote by $J$ the ideal of elements $v$ that satisfy (\#). Clearly $\left(I^{*} S+\mathbf{z} S\right) \subset J$. We claim that these ideals are actually equal. If not, take $v \in J$, that is, in the socle of $S /\left(I^{*} S+\mathbf{z} S\right)$. As in the proof of Theorem 3.3, we write $v=\sum s_{i} u_{i} b$, where $u_{i}, i=1, \ldots, n$ are generators for the socle of $R / I^{*}$. As in Theorem 3.3, we get that $c u_{i}^{Q} \in\left(I^{*}\right)^{[Q]}$. However, $c$ is a test element, and so $c^{2} u_{i}^{Q} \in I^{[Q]}$. Therefore, $u_{i} \in I^{*}$. So, $v \in I^{*} S$, which is a contradiction, and we are done. 


\section{REFERENCES}

[1] I. M. Aberbach, Tight closure in F-rational rings, Nagoya Math. J. 135 (1994), 43-54. MR 95g:13020

[2] I. M. Aberbach, Some conditions for the equivalence of weak and strong F-regularity, Comm. Algebra 30 (4) (2002), 1635-1651.

[3] I. M. Aberbach, Extension of weakly and strongly F-regular rings by flat maps, J. Algebra 241 (2) (2001), 799-807. MR 2002f:13008

[4] I. M. Aberbach, M. Hochster, and C. Huneke, Localization of tight closure and modules of finite phantom projective dimension, J. Reine Angew. Math. (Crelle's Journal) 434 (1993), 67-114. MR 94h:13005

[5] A. Bravo and K. E. Smith, Behavior of test ideals under smooth and étale homomorphisms, J. Algebra 247 (1) (2002), 78-94.

[6] W. Bruns and J. Herzog, Cohen-Macaulay rings, Cambridge University Press, Cambridge, 1998. MR 95h:13020

[7] F. Enescu, On the behavior of F-rational rings under flat base change, J. Algebra 233 (2000), 543-566. MR 2001j:13007

[8] M. Hashimoto, Cohen-Macaulay F-injective homomorphisms, Geometric and combinatorial aspects of commutative algebra (Messina, 1999), Lecture Notes in Pure and Appl. Math., 217, Marcel Dekker, New York, 2001, pp. 231-244. MR 2002d:13007

[9] M. Hochster and C. Huneke, Tight closure, invariant theory, and the Briançon-Skoda theorem, J. Amer. Math. Soc. 3 (1990), 31-116. MR 91g:13010

[10] M. Hochster and C. Huneke, Tight closure and elements of small order in integral extensions, J. Pure Appl. Algebra 71 (1991), 233-247. MR 92i:13002

[11] M. Hochster and C. Huneke, F-regularity, test elements, and smooth base change, Trans. Amer. Math. Soc. 346 (1994), 1-62. MR 95d:13007

[12] M. Hochster and C. Huneke, Localization and test exponents for tight closure (Dedicated to William Fulton on the occasion of his 60th birthday), Michigan Math. J. 48 (2000), 305-329. MR 2002a:13001

[13] C. Huneke, Tight closure and strong test ideals, J. Pure Appl. Algebra 122 (1997), 243250. MR 98g:13003

[14] E. Kunz, On Noetherian rings of characteristic p, Amer. J. Math. (1976), 999-1013. MR 55:5612

[15] S. Loepp and C. Rotthaus, Some results on tight closure and completion, J. Algebra 246 (2001), 859-880.

[16] G. Lyubeznik and K. E. Smith, On the commutation of the test ideal with localization and completion, Trans. Amer. Math. Soc. 353 (2001), 3149-3180. MR 2002f:13010

[17] H. Matsumura, Commutative ring theory, Cambridge University Press, Cambridge, 1986. MR 88h:13001

[18] J. Nishimura, A few examples of local rings, I, preprint, 1994.

[19] K. E. Smith, Test ideals in local rings, Trans. Amer. Math. Soc. 347 (1995), 3453-3472. MR 96c:13008

[20] J. Vélez, Openness of the F-rational locus and smooth base change, J. Algebra 172(2) (1995), 425-453. MR 96g:13003

[21] A. Vraciu, Strong test ideals, J. Pure Appl. Algebra 167 (2-3) (2002), 361-373.

Department of Mathematics, University of Missouri, Columbia, Missouri 65211

E-mail address: aberbach@math.missouri.edu

Department of Mathematics, University of Michigan, Ann Arbor, Michigan 48109

Institute of Mathematics of the Romanian Academy, Bucharest, Romania

E-mail address: fenescu@umich.edu

Current address: Department of Mathematics, University of Utah, Salt Lake City, Utah 84112 